\title{
Influenza Vaccine
}

\author{
JOSEPH E. SMADEL, M.D.
}

A No'THER significant episode in the mass immunization of a large civilian population occurred during the $A$ sian influenza epidemic. The acceptance of the current influenza vaccine program by laymen, physicians, and public health officials undoubtedly was farorably influenced by the highly successful campaigns for immunization against poliomyelitis. On the other hand, the development of $A$ sian influenza vaceine and the production of almost 50 million ml. of this vaccine during the 7 months after the newly isolated viral agent was first brought to this country represent a remarkable feat which could have been accomplished only because of the enormous experience gained through the extensive study of influenza during the past two decades.

I)r. Thomas Francis and his associates of the Commission on Influenza of the Armed Forces Epidemiological Board have been outstanding leaders in the extended studies of influenza and in the development and demonstration of the efficacy of influenza vaccines (1). Following the pattern developed by the commission, the Army Medical Department and the Public Health Service created a series of influenza watch laboratories to assist in providing prompt and accurate information on the occurrence and spread of influenza. 'These watch laboratories have since been integrated with the World Health Organization's system which now encircles the globe (.P).

Dr. Smadel is an associate director, National Institutes of Health, Bethesda, Md. This paper is based on comments at a symposium on Asian influenza held by the American College of Preventive Medicine and the health officers' section at the 1957 meeting of the American Public Health Association in Cleveland, Ohio.
A little-known experiment undertaken by Imerican influenza investigators and the vaccine producers several years ago provided additional background for the work in 1957 with Asian influenza vaccine. In 1951 England experienced a moderately severe outbreak of influenza, which was considered a possible hazard to the United States. It was decided to determine how quickly the British virus could be imported and analyzed for antigenic composition, and incorporated into a vaccine, if it were found to be different from the earlier strains of influenza virus. Accordingly, at the height of the epidemic in Liverpool, British colleagues supplied a strain which was flown to the Cnited States and promptly distributed to the laboratories at Walter Reed Army Institute of Research and the National Institute of Allergy and Infectious I)iseases, Public Health Service. The institute, in turn, sent samples to the influenzal vaccine manufacturers. 'The self-imposed problem of the various laboratories was to produce 1 liter of vaccine of acceptable potency, sterility, and safety in the shortest possible time. The two Government laboratories accomplished their task before the end of 3 weeks and the biologic houses shortly thereafter. The various workers recognized the portent of their achievement but realized that many factors had been in their favor and that similar success might not be regularly attained.

\section{Development of Asian Influenza Vaccine}

'The present influenza vaccine program must make obeisance to each of the earlier developments described above. Ising the watch laboratory organization, the Army Medical Department isolated the Asian influenza virus from patients in the Far East in late Ipril. 
By mid-May the Department recognized that the virus was antigenically dissimilar from other influenza $\mathrm{A}$ viruses and that the American population was virtually without protective antibodies against the new strain (3). Simultaneously, investigators in other countries made comparable observations. Numerous other investigators in university, governmental, and industrial laboratories in the United States began their studies on the Asian virus during the last week of May. In the meantime, WHO influenza detection centers throughout Asia, the Western Pacific, Australia, and the Middle East noted and reported the rapid spread of influenza from Hong Kong. It soon became apparent that the new virus was highly contagious, provoking large epidemics affecting up to 20 percent of the general population in one area after another, and in certain selected groups involving 60-70 percent of the population. Fortunately, the mortality remained low as the disease continued its sweep (4).

As a result of intensive work in the commercial laboratories, the first experimental lots of Asian monovalent vaccine were available before mid-June and were promptly placed under assay in volunteers by personnel of the Commission on Influenza, the Army, and the Public Health Service. It is worth noting that the first cases of Asian influenza were diagnosed in the continental United States during the first week of June $(5 a)$.

The military services, during the latter part of June, provided a most important stimulus to the commercial production of vaccine by inviting bids for 2,650,000 ml. of monovalent Asian vaccine. At about the same time, the Surgeon General of the Public Health Service informed the manufacturers that he anticipated the need for large amounts of influenza vaccine for use by the civilian population during the coming fall and winter. With these demonstrations of active interest in a vaccine prepared against the newly isolated Asian influenza virus, the manufacturers that had produced influenza vaccine in previous years offered to make a strenuous effort to meet the demands created by the anticipated epidemic. These manufacturers undertook this commitment, which involved considerable financial risk, without government subsidy.

\section{Vaccine Dosages}

The Public Health Service recommends tne following dosages of Asian influenza vaccine:

For those who have not been vaccinated, subcutaneous injection of $1.0 \mathrm{cc}$. of the $400 \mathrm{CCA}$ unit strength Asian influenza vaccine currently being produced.

For those who have received $0.1 \mathrm{cc}$. intracutaneously or $1.0 \mathrm{cc}$. subcutaneously of the 200 CCA unit vaccine previously produced and for those individuals at special risk (pregnant women, older persons, and those with chronic respiratory or cardiac disease), a second $1.0 \mathrm{cc}$. subcutaneous injection of 200 CCA vaccine is recommended. Physicians may also wish to recommend a second injection for other patients. If the $400 \mathrm{CCA}$ unit strength vaccine is available, 0.5 cc. should be given. The second dose should be received not less than 2 weeks after the first.

For children, the recommendation of the American Academy of Pediatrics is: For children under 5 years of age, 0.1 cc. intracutaneously or subcutaneously, repeated after 1 to 2 weeks; for children from 5 to 12 years of age, 0.5 cc. subcutaneously, repeated after an interval of 1 to 2 weeks; for children over age 13, the adult dosage.

The summer months were devoted to solving technical problems such as $(a)$ increasing the yield of virus growing in embryonated eggs, $(b)$ developing methods for assaying potency which would circumvent the time-consuming procedures previously employed, (c) determining the immunogenicity of the vaccine in man, $(d)$ training new technical teams in the pharmaceutical houses and renovating old facilities or constructing new, and (e) arranging for unprecedented quantities of fertile hens' eggs.

By mid-August, two manufacturers had produced a total of $500,000 \mathrm{ml}$. of monovalent Asian vaccine and obtained its release by the Division of Biologics Standards of the Public Health Service. Progress thereafter was rapid. Before the end of the first week in September, each of the six companies manufacturing the vaccine had produced one or more lots 
meeting the requirements. Moreover, $4,600,000$ ml. of vaccine (total bulk volume) was released by the Division of Biologics Standards by September 5. Production increased dramatically, and by the end of October $42,600,000 \mathrm{ml}$. bulk vaccine had passed the tests performed concurrently by the vaccine manufacturers and by the Division of Biologics Standards.

\section{Potency and Efficacy of Vaccine}

Drawing on the fund of knowledge accumulated over the years from field trials by the Commission on Influenza, it was estimated in June that the effective dose of Asian strain vaccine would probably be not less than 200 chicken cell agglutination (CCA) units when administered subcutaneously to adult human beings. Because of the difficulties encountered during the early summer in obtaining high yields of Asian virus in embryonated eggs, it seemed unlikely that a vaccine containing more than 200 CCA units of Asian antigen could be produced. Hence, the potency of the new monovalent vaccine was set at 200 CCA units per milliliter $(6 a)$.

A series of studies undertaken during the summer by members of the commission, the Army Medical Department, the Communicable Disease Center, and the National Institute of Allergy and Infectious Diseases was designed to determine the effective dose of Asian antigen. Only those experiments bearing on three specific points will be mentioned. One is concerned with the level of antibody which developed in patients convalescent from Asian influenza. Another deals with the amount of viral antigen required to elicit a similar level of antibody in a vaccinated person. A third point is concerned with the level of antibody found in volunteers who were resistant to experimental challenge with Asian virus.

If some license is permitted in interpreting data of others, it may be stated that levels of hemagglutination inhibition antibodies of the order of a titer of 1:32 actual dilution of serum (or 1:128 final dilution of serum) were present in convalescent serums (3). Moreover, to induce a similar level in about 90 percent of persons inoculated with a single dose of vaccine given subcutaneously, 400-500 CCA units of antigen were required ( $(y)$, according to Keith E. Jensen of the Communicable Disease Center. Finally, appreciable resistance to challenge was displayed by those volunteers who had antibody levels of the order of 1:32. (In the tests of Bell and associates ( $y), 78$ percent of the placebo controls developed signs of illness following challenge; 60 percent of vaccinated persons who failed to acquire detectable antibodies became ill; 43 percent of those with titers of $1: 10$ to $1: 20$ developed influenza; while only 25 percent of those who had titers of $1: 40$ or greater became ill.)

Such information as this, coupled with knowledge of the improved yields of virus from infected eggs and the increased facilities of the manufacturers, led to a recommendation in October that the potency of monovalent Asian influenza vaccine "be increased from 200 CCA units to 400 CCA units per milliliter as soon as feasible and not later than December 1, 1957" $(6 b)$. Current recommendations for the use of the vaccine appear on p. 130.

October 1957 saw the shift to the production of 400 CCA unit vaccine. Indeed, by November 1, two manufacturers had submitted to the Division of Biologics Standards for concurrent testing 13 lots with the $400 \mathrm{CCA}$ unitage. As of November 12, 2 million ml. of $40 \theta$ unit vaccine had been released by the division; 13 million ml. was available by December 12 .

The report of Dr. Fred Davenport which appears in this issue (pp. 133-139) presents the available field information dealing with efficacy of Asian vaccine among military populations. In general, the data provide grounds for encouragement.

For many years, the influenza vaccines used in civilian and military populations have been of the polyvalent type, containing several strains of influenza $A$ virus and one or more strains of influenza $B$ virus. The formula for a polyvalent material which is now being produced for use during the latter part of the winter is as follows: influenza A, Asian strain200 CCA; influenza A', PR 301 strain-100 CCA ; influenza A, PR 8 strain-100 CCA ; and influenza B, Great Lakes strain-100 CCA. The recommended basic course of immunization consists of 2 subcutaneous injections each 
of $1.0 \mathrm{ml}$., the second following the first by not less than 2 weeks.

\section{Future}

While the record to date for developing and producing Asian vacine has been truly amazing, it would be foolhardy to assume that it will be possible to do as well or better the next time the country is confronted with a severe epidemic caused by an unfamiliar strain of influenza virus. There was an interval of almost 6 months between the time the first strain of Asian virus reached a research laboratory in the United States and the time at which the weekly pneumonia and influenza deaths increased above the "epidemic threshold" in the 108 cities which are under surveillance by the Communicable Disease Center (5b).

Suppose the next widespread occurrence of influenza begins in North America or arrives here promptly and reaches epidemic proportions within a month or so? The measures which were successful in this epidemic did not provide any useful quantity of vaccine during the first 2 months after the virus seed reached the manufacturers. I think it is obvious that the search must continue for means of rapidly increasing the resistance of populations to epidemic influenza.

Recognition of this fact was one of the reasons the Surgeon General of the Public Health
Service, on the reconmendation of the Association of State and Territorial Health Officers, established an Advisory Committee on Influenza Research. This committee was asked, among other things, to consider and advise the Surgeon General on what further research might be undertaken to strengthen influenza control in the future and to suggest where such research might most profitably be done.

\section{REFERENCES}

(1) Francis, T., Jr.: Vaccination against influenza. Bull. World Health Org. 8: 725-744 (1953).

(2) I aris, I). J.: World Health Organization influenza study program in the United States. I'ub. Health Rep. 67: 1185-1190, December 1952.

(3) Meyer, H. M., Jr., Hilleman, M. R., Miesse, M. I., Crawford, I. P., and Bankhead, A. S.: New antigenic variant in Far East influenza epidemic, 19:77. I'roc. Soc. Exper. Biol. \& Med. 95 : $609-616$ (1957).

(4) The 1957 influenza epidemic. Chron. World Health Org. 11:269-271 (1957).

(j) U. S. Communicable Disease Center: Influenza surveillance report (a) No. 1, July 9; (b) No. 22, Oct. 21. Atlanta, Ga., 1957.

(6) Murray, R.: Division of Biologics Standards memorandum to manufacturers of influenza vaccine (a) July 2, (b) Oct. 9. Bethesda, Ma., 1957.

( $)$ Bell, J. A., Ward, T. G., Kapikian, A. Z., Shelokov, A., Reichelderfer, T. E., and Huebner, R. J.: Artificially induced Asian influenza in vaccinated and unvaccinated volunteers. J. A. M. A. 165: 1366-1373 (1957).

\section{Vaccines, 1919}

"The only way in which we are to secure promptly acceptable evidence of the value of a bacterial vaccine is by the vaccination of only a portion of the individuals in a large group, holding the remainder as controls; age, sex, and conditions of exposure being the same in the two groups.

"On the other hand, a vaccine should not be condemned unless controlled as just indicated, and unless it has failed to show protec- tive value when sufficient time has elapsed after the inoculation to make it reasonably likely that any immunity which may develop will have had an opportunity to do so.

"A large number of vaccines have been used, some made from the influenza bacillus alone, others from this in conjunction with pneumococci, staphylococci, and streptococci, and in various combinations; the failure of one does not necessarily mean the uselessness of others."

-G. W. McCoy, Director, Hygienic Laboratory, Public Health Service, Public Health Reports, May 30, 1919. 